\title{
On The Effects of Column Occupancy and Static Atomic Disorder on the Analysis of Chemical Ordering in $\left.\mathbf{G a}\left(\mathbf{P}_{(1-\mathrm{x})}\right) \mathbf{B i}_{\mathbf{x}}\right)$ Compounds
}

\author{
Jürgen Belz ${ }^{1}$, Andreas Beyer ${ }^{1}$, Lukas Nattermann $^{1}$ and Kerstin Volz ${ }^{1}$ \\ 1. Materials Science Center and Faculty of Physics, Philipps-Universität Marburg, Marburg, Germany
}

Dilute bismides have been considered promising materials for new highly efficient lasers for telecommunication applications due to their reduced Auger recombination [1]. Since electronic calculations can depend on ordering effects [2], the following work is aimed towards the characterization of clustering behavior of bismuth in gallium phosphide bismide $(\mathrm{Ga}(\mathrm{PBi})$ ) by scanning electron transmission microscopy (STEM). Hereto, we investigate the effects of static atomic disorder (SAD) on the intensity distribution in such a material as well as the implications of intra-column distributions of bismuth with respect to the scattered intensity.

The compound has been grown pseudomorphically on gallium phosphide $(\mathrm{GaP})$ substrate by metal organic vapor phase epitaxy. A test structure containing multiple layers of $\mathrm{Ga}(\mathrm{PBi})$ with different bismuth fractions and separated by GaP layers is thinned along the [010] direction by broad argon ion beam milling. The microscopy was carried out with a double aberration corrected JEOL JEM-2200FS at $200 \mathrm{kV}$ in scanning mode using an annular dark field detector (ADF).

Upon investigation, the bismuth containing layers possess a remarkable appearance when investigated with STEM due to the highly different scattering power of bismuth (atomic number $Z=83$ ) compared to that of phosphorous $(Z=15)$. Since both elements occupy the group $V$ sub-lattice in the zinc blende structure, the overall image contrast is very significantly altered by small amounts of bismuth (fig. 1a).

In order to characterize the alloy with respect to ordering on the atomic scale by STEM measurements image simulations are carried out first. We hereto utilize our newly developed STEMsalabim code [3] in order to perform accurate multi slice simulations using the frozen lattice approach as well as modelling the probe of an aberration corrected STEM including the $\mathrm{C}_{\mathrm{C}}$ induced defocus spread.

The impact of SADs is evaluated by the comparison of simulated STEM ADF intensities of rigid and valance force field relaxed supercells. It can be seen that on-column intensities are redistributed from every ADF scattering range into the diffuse background with a bias towards lower scattering angles. This finding can be directly correlated with the distortion of gallium position when they are neighboured by bismuth atoms.

On the other hand, in experimental images (cf. fig. 1a) gallium columns in the alloy show up to $15 \%$ higher intensities than those of the neighbouring undistorted binary material which is at first glance contrary to the aforementioned simulation results. Upon further investigation it can be concluded that the drastic increase of these intensities has to result from the huge scattering power of even single bismuth atoms and the resulting intensity overlap with the gallium columns.

The effect of intensity crosstalk is therefore considered in a systematic image simulation study. Hereto, we model a large supercell of pure $\mathrm{GaP}$ and substitute single phosphorous atoms (separated by one unit cell) with bismuth atoms at different z-positions along the electron beam direction. It can be seen that the 
depth measured from the top surface is of great relevance when considering the distribution of bismuth in GaP. We show that when one atom lies in the vicinity of the top surface its scatter appears up to three times as intense as compared to a deep $(>15 \mathrm{~nm})$ region, and that such an atom contributes a significant amount of scattered intensity to even the $2^{\text {nd }}$ nearest neighbouring column.

Finally, an image simulation from a randomly ordered $\mathrm{Ga}\left(\mathrm{P}_{96.8 \%} \mathrm{Bi}_{3.2 \%}\right)$ supercell is used to derive the apparent number of bismuth atoms from the averaged group V on-column (fig. 1b). Here we can identify a region (red box) where intense columns (pointing to a high bismuth occupancy) are neighboured by other bismuth-rich columns. Nevertheless, this effect can be shown to be partially an artefact as described before when considering the actual supercell occupation (fig. 1c). In addition, we can find very bright columns (purple boxes) where very few bismuth atoms are present and only the central column intensity is high. This finding can be correlated with the large difference of the z-position of bismuth atoms within these neighbouring columns.

We can show that the effects of SADs, z-position and column crosstalk are of great relevance when considering atomic ordering analysis in $\mathrm{Ga}(\mathrm{PBi})$ using STEM. These complex effects have to be accounted for when deriving an accurate distribution model for bismuth in $\mathrm{GaP}$ on an atomic scale [4].

\section{References:}

[1] Polak, M. P., P. Scharoch, and R. Kudrawiec, Semicond. Sci. Technol. 30.9 (2015) p. 094001.

[2] Franceschetti et al, Physical Review B 52.19 (1995) p. 13992.

[3] J.O. Oelerich et al, Submitt. Publ. (2016). http://www.online.uni-marburg.de/stemsalabim/.

[4] The authors acknowledge support from the German Science Foundation (GRK 1782:

"Functionalization of Semiconductors") and the German Federal Ministry of Education and Research through the project "MehrSi".
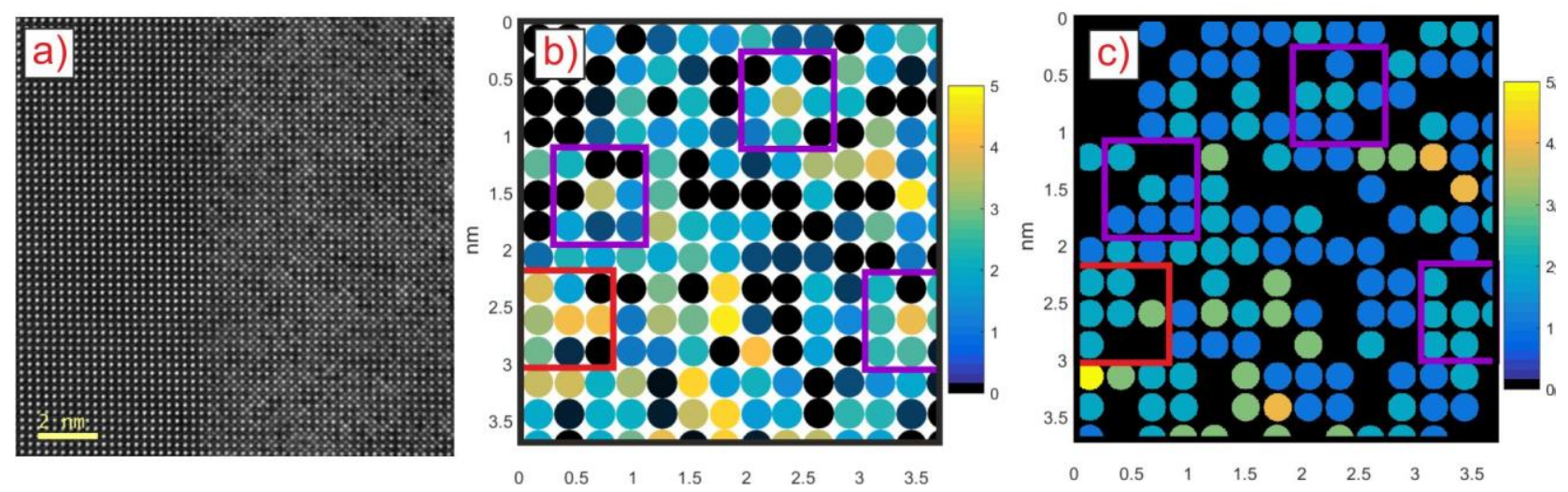

Figure 1. (a) Experimental STEM image of a $\mathrm{GaP} / \mathrm{Ga}\left(\mathrm{P}_{96.8 \%} \mathrm{Bi}_{3.2 \%}\right)$ interface. (b) Bismuth occupancy derived from an image simulation of a supercell containing 3.2\% bismuth (group V columns shown). (c) The actual supercell composition is shown in where atoms at the surface have high intensity and can raise the local intensity environment. 